\title{
Chemical Transformation of Carboxyl Groups on the Surface of Silicon Carbide Quantum Dots
}

Zsolt Szekrényes, Bálint Somogyi, Dávid Beke, Gyula

Károlyházy, István Balogh, Katalin Kamarás, and Adam Gali

J. Phys. Chem. C, Just Accepted Manuscript • DOI: 10.1021/jp5053024 • Publication Date (Web): 08 Aug 2014

Downloaded from http://pubs.acs.org on August 12, 2014

\section{Just Accepted}

"Just Accepted" manuscripts have been peer-reviewed and accepted for publication. They are posted online prior to technical editing, formatting for publication and author proofing. The American Chemical Society provides "Just Accepted" as a free service to the research community to expedite the dissemination of scientific material as soon as possible after acceptance. "Just Accepted" manuscripts appear in full in PDF format accompanied by an HTML abstract. "Just Accepted" manuscripts have been fully peer reviewed, but should not be considered the official version of record. They are accessible to all readers and citable by the Digital Object Identifier (DOI®). "Just Accepted" is an optional service offered to authors. Therefore, the "Just Accepted" Web site may not include all articles that will be published in the journal. After a manuscript is technically edited and formatted, it will be removed from the "Just Accepted" Web site and published as an ASAP article. Note that technical editing may introduce minor changes to the manuscript text and/or graphics which could affect content, and all legal disclaimers and ethical guidelines that apply to the journal pertain. ACS cannot be held responsible for errors or consequences arising from the use of information contained in these "Just Accepted" manuscripts. 


\title{
Chemical Transformation of Carboxyl Groups on the Surface of Silicon Carbide Quantum Dots
}

\author{
Zsolt Szekrényes, ${ }^{\dagger}$ Bálint Somogyi, ${ }^{\dagger, \ddagger}$ Dávid Beke, ${ }^{\dagger}$, Gyula Károlyházy, ${ }^{\dagger, \uparrow}$ István \\ Balogh, ${ }^{\dagger}$ Katalin Kamarás, ${ }^{\dagger}$ and Adam Gali*,,+† \\ Wigner Research Centre for Physics, Institute for Solid State Physics and Optics, \\ Hungarian Academy of Sciences, H-1525 Budapest, Hungary, Department of Atomic \\ Physics, Budapest University of Technology and Economics, H-1111, Budapest, Hungary, \\ and Faculty of Chemical Technology and Biotechnology, Budapest University of Technology \\ and Economics, H-1111 Budapest, Hungary \\ E-mail: gali.adam@wigner.mta.hu
}

\begin{abstract}
Silicon carbide quantum dots in the size range of $1-10 \mathrm{~nm}$ are in the center of interest with unique properties that makes them very promising biomarkers. A central requirement for this application is the control over the complex structure of the surface to enable further surface functionalization processes, which are crucial for drug delivery. In this paper a temperature dependent infrared and photoluminescence spectroscopy study, combined with ab initio modeling, is presented in order to reveal the chemical transformations of the surface termination groups. We found that at temperatures above $370 \mathrm{~K}$ acid anhydride groups form by condensation of water between neighboring

*To whom correspondence should be addressed

${ }^{\dagger}$ Wigner Research Centre for Physics

†Department of Atomic Physics, Budapest University of Technology and Economics

『Faculty of Chemical Technology and Biotechnology, Budapest University of Technology and Economics


carboxyl groups. The presence of the anhydride groups reveals the proximity of the carboxyl groups, and represents a new possibility of selective engineering of new hybrid materials involving silicon carbide quantum dots.

\section{Introduction}

Silicon carbide quantum dots ( $\mathrm{SiC}$ QDs) are known to be very promising structures for bioimaging, as well as optoelectronic applications, because of the superior properties of the bulk material. ${ }^{1-7}$ Silicon carbide is a wide band gap semiconductor with excellent hardness and chemical resistivity ${ }^{5}$ and is also known as a bioinert material. ${ }^{8-10}$ Depending on the starting bulk powder, the surface of $\mathrm{SiC}$ QDs is often rich in various functional groups which can result in diverse behaviors in biological environments ranging from bioinertness to changes in cell function and cytotoxicity. ${ }^{3,11}$ While the successful application of the $\mathrm{SiC}$ QDs in bioimaging techniques is related to their bioinert and photostable properties, ${ }^{2,6}$ further applications in medicine and drug delivery rely on the ability of engineering the desired surface properties by attaching different functional molecular groups. To obtain tailor-made functionalized surfaces it is necessary to understand the complex structure of the QD surface. There are several experimental and theoretical studies in the literature about the surface chemistry of $\mathrm{SiC}$ QDs where the presence of $\mathrm{Si}-\mathrm{O}-\mathrm{Si}, \mathrm{C}-\mathrm{O}-\mathrm{C}, \mathrm{C}=\mathrm{O}$, and -OH groups was observed. ${ }^{1,2,12-18}$ Photoluminescent properties of the SiC QDs, as those of other QDs, are greatly influenced by their surface chemical structure as some surface radicals can form new energy levels in the band gap and can act as new radiative centers. ${ }^{17,18}$ Even though some studies explain optical properties of SiC QDs by the existence and dissociation of hydroxyl groups, clear evidence of $\mathrm{Si}-\mathrm{OH}$ or $\mathrm{C}-\mathrm{OH}$ terminations is still absent because of the complex vibrational region above $3000 \mathrm{~cm}^{-1}$, where vibrations of adsorbed water overlap with the surface related -OH vibrational bands. Experiments concerning the solvent polarity dependence of the photoluminescence (PL) of SiC QDs yielded conflicting results in the literature. While Zakharko et al. ${ }^{19}$ measured a red-shift with decreasing solvent polarity 
in the PL emission, Chu et al. ${ }^{20}$ found the opposite trend, a redshift with increasing solvent polarity. The importance of understanding and controlling the surface structure is also significant from the point of view of $\mathrm{pH}$ sensitivity. ${ }^{11}$ In our previously published results ${ }^{1}$ we observed similar dependence as Chu et al. ${ }^{20}$ and assign these different behaviors to differences related to the synthesis procedures which can yield different surface structures. One possible explanation for the different physical and chemical properties of similar SiC QDs solutions is related to the diversity in surface terminations which can be related to the variations of the carboxyl concentration or the amount of Si on the surface. On the other hand, surface modification was reported only through the reaction of carboxyl groups which usually do not constitute the dominant part of the surface.

Here we report a study on $\mathrm{SiC}$ QDs based on $\mathrm{SiC}$ powder from two different sources (one synthesized in our laboratory and one commercial). We found that the surface structure is highly sensitive to the starting $\mathrm{SiC}$ powder properties like grain size and porosity (based on previously published results the source $\mathrm{SiC}$ grain size is in the range of 10-20 $\mu \mathrm{m}$ and possesses properties which are close to the bulk SiC properties ${ }^{1}$ ). There are important differences in the infrared spectra of the studied samples: we observed a more dominant carboxylic $\mathrm{C}=\mathrm{O}$ vibrational band in the sample prepared from $\mathrm{SiC}$ powder synthesized in our laboratory (sample 1) than in the sample prepared from commercial SiC powder (sample 2). We performed a temperature dependent investigation from room temperature up to $450 \mathrm{~K}$ to follow the effect of dehydration and to get extra information on the surface and the carboxyl group transformations. We followed these processes by infrared (IR) and photoluminescence (PL) spectroscopy and found clear evidence of acid anhydride formation from carboxyl groups above $370 \mathrm{~K}$. Our ab initio modeling on these processes supports the correlation between IR and PL properties as a function of surface termination. At higher temperatures the effect of dehydration was observed by a strong shift of the $-\mathrm{OH}$ vibration band (above $3000 \mathrm{~cm}^{-1}$ ) from a hydrogen bonded state to a free state. The free - $\mathrm{OH}$ vibrational band above 3600 $\mathrm{cm}^{-1}$ explicitly proves the presence of hydroxyl termination sites on the surface. 


\section{Materials and methods}

\section{Materials and experimental methods}

We prepared SiC QDs using top-down method ${ }^{1}$ from $\mathrm{SiC}$ powder synthesized in our laboratory reacting silicon powder (Acros Organics, mesh 325) and carbon black (Norit A supra, surface area $\left.(\mathrm{BET})-1700 \mathrm{~m}^{2} / \mathrm{g}\right)$. The reaction was conducted in an induction chamber in the presence of (poly)tetrafluoroethylene powder (Acros Organics, mesh 200) acting as activator. ${ }^{21}$ In this reaction $\mathrm{SiC}$ keeps the structure of the carbon black ${ }^{22}$ which explains its highly porous structure (sample 1). In another reaction, we synthesized SiC QDs from commercial SiC powder (US-Research Nanomaterials Inc.) with grain size in the range 1-40 $\mu \mathrm{m}$ (sample 2 ).

Colloidal SiC QD samples for the infrared measurements were prepared by drop-drying on the surface of a ZnSe crystal (crystal size 100x10x10 mm) and measured in attenuated total internal reflection (ATR) mode. The crystal geometry enabled multiple internal reflections to ensure high sensitivity for the detection of the weak surface termination groups at very small sample quantity. Temperature dependence was measured in transmission mode on SiC QDs dried on 10x10 mm clean Si wafer. Spectra were taken by various spectrometers: a Bruker IFS 66v and a Bruker Tensor 37 with $4 \mathrm{~cm}^{-1}$ resolution and DTGS detector. Spectra were recorded in the 400-4000 $\mathrm{cm}^{-1}$ range, except in the ATR mode where due to the multiple internal reflections the multi-phonon modes of ZnSe crystal absorb IR light below $700 \mathrm{~cm}^{-1}$. The baseline was corrected by an adjusted polynomial function.

Fluorescence spectra were recorded with a HORIBA Jobin Yvon Fluorolog-3 spectrofluorometer (Nanolog). The samples were measured in colloidal aqueous solution (concentration approx. $0.5 \mathrm{mg} / \mathrm{ml}$ ). The integration time was set to $0.1 \mathrm{~s}$ and the excitation wavelength was $320 \mathrm{~nm}$. 


\section{Theoretical methods}

We performed our calculations on a small-sized, spherical SiC QD containing 79 Si and $68 \mathrm{C}$ atoms with diameter of $1.4 \mathrm{~nm}$. This is a relatively small $\mathrm{SiC}$ QD that appears in our fabricated samples, ${ }^{2}$ nevertheless, we only aim to provide trends and semiquantitative results. The dangling bonds on the surface were terminated by hydrogen atoms. While this model is not realistic since the $\mathrm{Si}-\mathrm{H}$ bonds are not stable, it allowed us to concentrate solely on the effect of the carboxyl and anhydride groups on the QD surface. Although the Si atoms are likely to become oxidized, these defects do not influence the optical properties. ${ }^{17,23}$ However, we found that the defect level of $\mathrm{COO}^{-}$can hybridize with the neighboring Si-H bonds, which may lead to unrealistic results. To avoid this undesirable scenario, we replaced the hydrogen atoms with Si-OH and Si-O-Si structures in the vicinity of surface defects (Fig. 1).

The geometry optimizations were carried out within plane wave supercell formalism by using the $\mathrm{PBE}^{24}$ exchange-correlation functional within density functional theory (DFT). We used a $420 \mathrm{eV}$ and $1260 \mathrm{eV}$ cutoff for the wavefunction and charge density expansions, respectively. We applied standard PAW-potentials ${ }^{25}$ for the ions as implemented in the VASP code. ${ }^{26}$ The distance between any surface atoms of the periodic images was larger than $1 \mathrm{~nm}$, thus the interaction between the QDs was negligible. The geometry of the QDs was optimized until all force acting on the atoms fell below $0.02 \mathrm{eV} / \AA$. This criterion is sufficient for the accurate description of the electronic properties of QDs. The geometries of QDs selected for vibration calculations were further optimized until the force on each atom was smaller than $0.001 \mathrm{eV} / \AA$, in order to obtain accurate vibration frequencies. The vibrational spectra were calculated utilizing the density functional perturbation theory (DFPT) ${ }^{27}$ as it is implemented in the VASP code.

Excitation energies were calculated by time-dependent density functional (TDDFT) calculations by using TURBOMOLE cluster $\operatorname{code}^{28}$ where the optimized geometries were provided from the VASP calculations. We used a double- $\zeta$ polarized basis set which has 
proven to be sufficient ${ }^{29}$ for the accurate description of ground state properties and low energy excitations. We applied the PBE0 hybrid functional ${ }^{30,31}$ in the TDDFT kernel, which provides reliable results for both $\mathrm{SiC} \mathrm{QDs}^{23}$ and organic molecules. ${ }^{32}$ TDDFT calculations revealed that the excited state can be simply described by promoting a single electron from the HOMO to the LUMO.

For the calculation of the emission energies, we applied the Franck-Condon approximation. ${ }^{33}$ The potential energy surface of the ground and excited electronic states may differ, so the nuclear coordinates of the ground $\left(\mathbf{R}_{i}^{\mathrm{GS}}\right)$ and excited $\left(\mathbf{R}_{i}^{\mathrm{ES}}\right)$ states belonging to the minimum energy of their potential energy surfaces are also different. The geometry corresponding to the minimum energy of the potential energy surface in the excited state electronic configuration was determined by the $\triangle \mathrm{SCF}$ method, which is an efficient and rather reliable tool for such calculations ${ }^{34}$ when the excitation can be simply described by promoting a single electron from an occupied state to an empty state. The vertical emission energies were calculated by the application of the TDDFT method in $\mathbf{R}_{i}^{\mathrm{ES}}$ nuclei coordinates while the zero-phonon line $(\mathrm{ZPL})$ energy $\left(\epsilon_{\mathrm{ZPL}}\right)$ was derived as the sum of the vertical emission energy $\left(\epsilon_{\text {vert }}\right)$ and the total energy $\left(E_{\text {total }}\right)$ difference between the electronic ground states calculated by PBE0 functional in the ground and excited state geometries: $\epsilon_{\mathrm{ZPL}}=\epsilon_{\mathrm{vert}}\left\{\mathbf{R}_{i}^{\mathrm{ES}}\right\}+\left(E_{\text {total }}\left\{\mathbf{R}_{i}^{\mathrm{ES}}\right\}-E_{\mathrm{total}}\left\{\mathbf{R}_{i}^{\mathrm{GS}}\right\}\right)$.

\section{Results and discussion}

\section{Temperature dependence}

Figure 2 presents the infrared spectra measured at room temperature for sample $\mathbf{1}$ and sample 2. The band at approx. $800 \mathrm{~cm}^{-1}$ is assigned to $\mathrm{SiC}$. The featureless broad band centered at $1100 \mathrm{~cm}^{-1}$ is assigned to $\mathrm{C}-\mathrm{O}-\mathrm{C}$ and Si-O-Si vibrations. The most important region is located at $1720 \mathrm{~cm}^{-1}$ and is the $\mathrm{C}=\mathrm{O}$ vibration of the $\mathrm{COOH}$ group. This is also the molecular group which represents the main interest for further functionalization. Taking

\section{ACS Paragon Plus Environment}




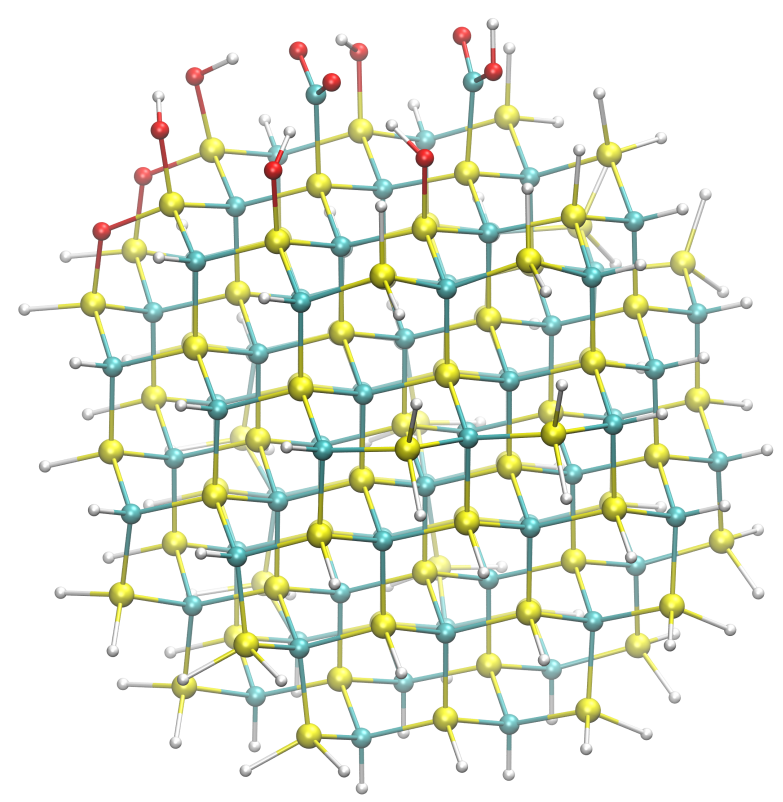

(a)

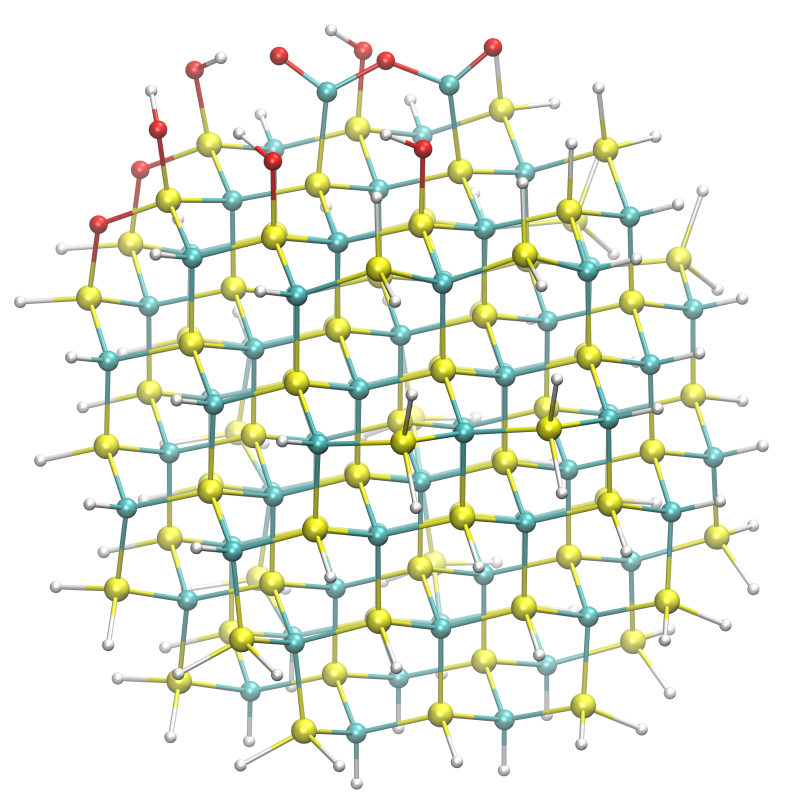

(b)

Figure 1: Ball and stick geometries of the two considered surface groups. (a) Si-COOH group near a $\mathrm{Si}_{-} \mathrm{COO}^{-}$group, (b) an anhydride group bonding to two $\mathrm{Si}$ atoms. For a realistic description of the surface, the $\mathrm{Si}-\mathrm{H}$ bonds close to the defects were replaced by $\mathrm{Si}-\mathrm{OH}$ groups and Si-O-Si bridges. White, cyan, yellow and red balls depict H, C, Si and O atoms, respectively. 
into account the relative intensity ratio between the oxide band and the carbonyl band we estimate a higher carboxyl concentration for sample $\mathbf{1}$ in comparison with sample $\mathbf{2}$. Above $3000 \mathrm{~cm}^{-1}$ the spectra are dominated by a broad $-\mathrm{OH}$ band assigned to the hydrogen bonded -OH and the hydrate shell around the QDs. Figure 3 (a) and (b) present the temperature

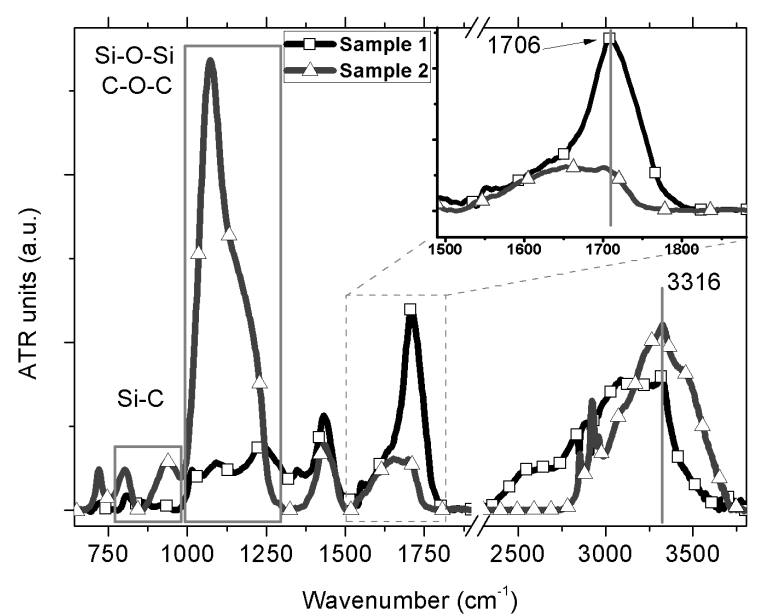

Figure 2: Infrared spectra of sample $\mathbf{1}$ (black line with empty squares) and sample $\mathbf{2}$ (grey line with empty triangles). There is a clear difference in the $\mathrm{C}=\mathrm{O}$ bands of the carboxyl group at $1720 \mathrm{~cm}^{-1}$ as well as in the region between $1000-1300 \mathrm{~cm}^{-1}$.

dependent infrared spectra of sample $\mathbf{1}$ and $\mathbf{2}$, respectively. Interesting behavior occurs at elevated temperatures. Two new bands appear at 1792 and $1860 \mathrm{~cm}^{-1}$ as the $\mathrm{C}=\mathrm{O}$ band is decreasing in intensity. This doublet band is characteristic of the acid anhydride $\mathrm{C}=\mathrm{O}$ vibrations. ${ }^{35}$ Additional information comes from the temperature dependence of the hydration shell related -OH band. According to figure 3 (a) and (b) anhydride formation is observed from $370 \mathrm{~K}$ whereas no drastic changes occur above $3000 \mathrm{~cm}^{-1}$ (water related -OH band). Above $400 \mathrm{~K}$ the carboxyl-carboxyl pair to anhydride transformation saturates as the intensity of the anhydride related bands becomes constant. The very broad -OH band between $3000-3600 \mathrm{~cm}^{-1}$ also shows a very strong temperature dependence. Above $400 \mathrm{~K}$ the decrease in intensity and narrowing of this broad band is getting more evident and is assigned to the complete dehydration of the SiC QDs. Similar transformations are observed also for sample $\mathbf{2}$ where the carboxyl concentration was supposed to be much lower. This 
behavior suggests that carboxyl sites should be in close proximity both in the high and low concentration situation. We consider two possibilities for the process of carboxyl to anhydride transformation:

(i) anhydride formation between two different SiC QDs leading to an inter-dot anhydride. This situation would be possible if the inter-dot carboxyl-carboxyl coupling through hydrogen bonds would be dominant during the drying process. The bound water evaporation and anhydride formation should occur simultaneously. According to figure 3 we conclude that this situation may not be probable as we observe the saturation of the anhydride formation process well before the bound water evaporation.

(ii) on-dot anhydride formation by water elimination between two neighboring carboxyl groups. If the synthesis process can favor carboxyl group formation (e.g., during the porous carbide formation where local charges play an important role, or during sonication when the weakly interconnected nanocrystallites are broken ${ }^{36}$ ) then the neighboring carboxyl sites can form anhydride groups. On-dot anhydride formation requires the presence of carboxyl groups in close proximity to each other and would require that at least one of the two carboxyl sites is not hydrogen bonded. ${ }^{37}$ The presumably short lifetime of a hydrogen bonded COOH above $370 \mathrm{~K}$ would ensure that unbounded carboxylic groups are available. ${ }^{38}$

The nature of the interaction between on-dot surface sites merits a detailed investigation. The $\mathrm{OH}$ and $\mathrm{COOH}$ groups on the $\mathrm{SiC}$ QDs surfaces interact with the water molecules of the solvent by hydrogen bonding. ${ }^{39}$ The existence of hydrogen bonding between neighboring $\mathrm{COOH}$ is excluded due to steric effects and proved by the absence of anhydride formation at room temperature in vacuum (as the situation in figure 2). At $450 \mathrm{~K}$ a distinct band emerges above $3600 \mathrm{~cm}^{-1}$ which is characteristic of the free $-\mathrm{OH}$ group. As the anhydride formation is complete at this temperature, the presence of $\mathrm{COOH}$-related $\mathrm{OH}$ groups is less probable. We assign this band to $\mathrm{Si}-\mathrm{OH}$ and $\mathrm{C}-\mathrm{OH}$ hydroxyl groups which are present also at room temperature at the surface together with $\mathrm{COOH}$ groups. As shown in figure 3 (a) and (b), after anhydride formation the -OH groups are still mostly in hydrogen bonded structure 


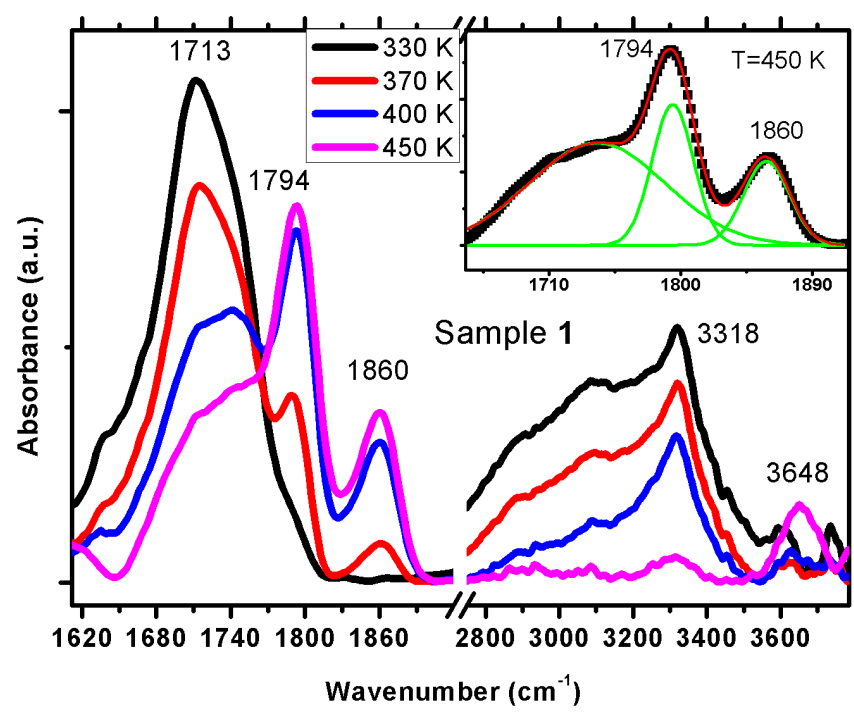

(a)

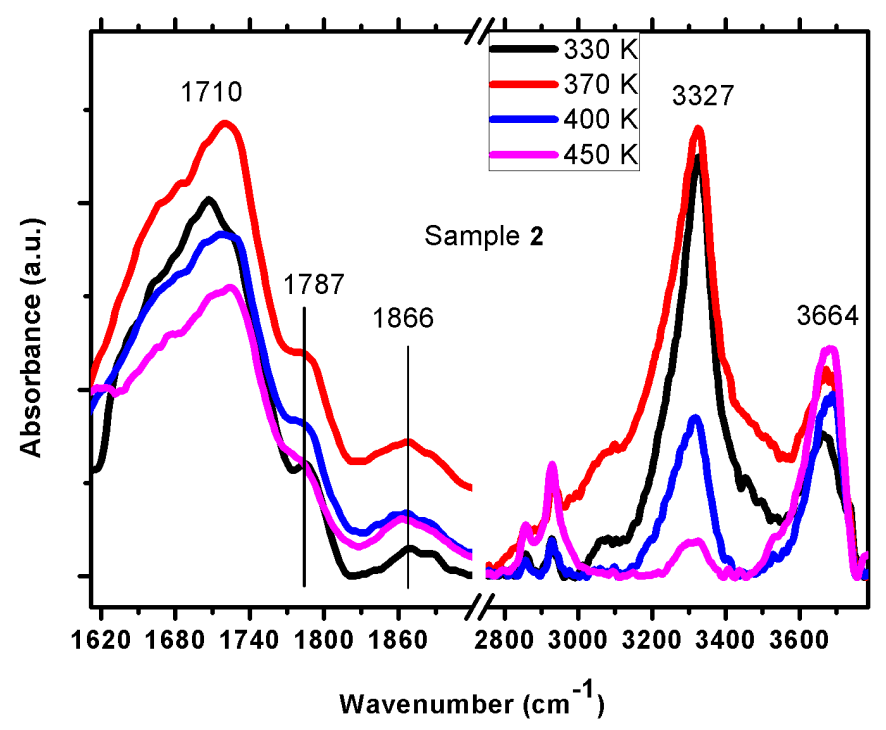

(b)

Figure 3: Temperature dependent infrared spectra of sample 1 (a) and sample $\mathbf{2}$ (b). Above $370 \mathrm{~K}$ there is a strong decrease in intensity of the carboxylic $\mathrm{C}=\mathrm{O}$ band $\left(1720 \mathrm{~cm}^{-1}\right)$ and a doublet band characteristic of the anhydride group appears at 1792 and at $1860 \mathrm{~cm}^{-1}$. The strong decrease in intensity of the $\mathrm{O}-\mathrm{H}$ band above $3000 \mathrm{~cm}^{-1}$ is assigned to the evaporation of the hydrate shell. Inset in (a): Gaussian fit to the IR spectrum taken at $450 \mathrm{~K}$ in the $\mathrm{C}=\mathrm{O}$ vibration region. Single Gaussian bands situated at $1794 \mathrm{~cm}^{-1}$ and at $1860 \mathrm{~cm}^{-1}$ indicate the formation of anhydride groups on the $\mathrm{Si}$ side of $\mathrm{SiC}$ QDs. 
with water molecules (broad band above $3000 \mathrm{~cm}^{-1}$ ). This means that the hydrogen bonds between the water molecules and hydroxyl termination sites are stronger than the hydrogen bonds with carboxyl groups, even though in organic molecules carboxylic acids form much stronger hydrogen bonds than alcohols. Similar results were obtained on nanodiamond (ND) surfaces - water interaction where it was measured that the hydrogen bonds between carboxyl terminated NDs $(\mathrm{C}-\mathrm{COOH})$ and water molecules are weaker than the hydrogen bonds between hydroxyl terminated NDs and water molecules. ${ }^{39}$
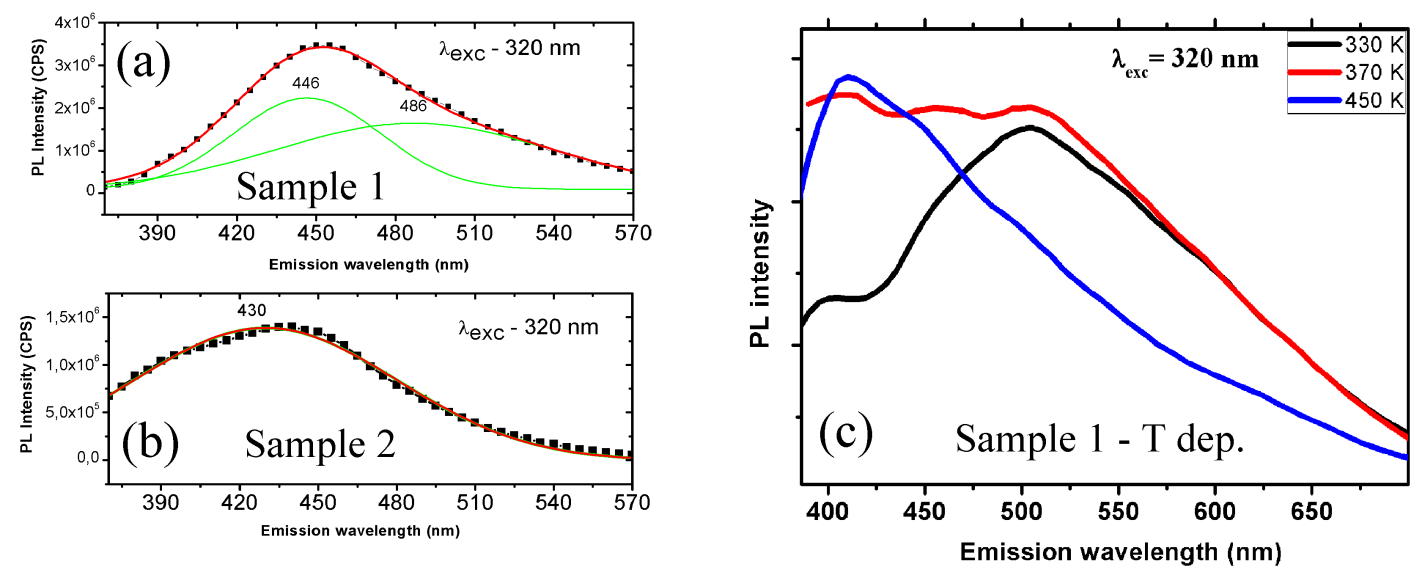

Figure 4: Photoluminescence spectra of (a) sample $\mathbf{1}$ and (b) sample $\mathbf{2}$ recorded in water at the maximum emission line with $320 \mathrm{~nm}$ excitation wavelength. (c) presents the temperature dependent photoluminescence spectra of sample 1 recorded in solid form of $\mathrm{SiC}$ QDs on silicon wafer recorded after water evaporation at $330 \mathrm{~K}$. The main emission band is located around $500 \mathrm{~nm}$, at $370 \mathrm{~K}$ a clear transition is observed to lower wavelengths, indicating important changes on the surface of $\mathrm{SiC}$ QDs, and at $450 \mathrm{~K}$ the emission maximum is close to $400 \mathrm{~nm}$. In this temperature region bound water has evaporated and anhydride functional groups are formed.

We studied the effect of anhydride formation also on the PL properties of SiC QDs. Figure 4 (a) and (b) show the PL spectra of sample 1 and sample 2 recorded at $320 \mathrm{~nm}$ excitation. The origin of the emission bands is assigned to a complex contribution of the SiC QDs LUMO-HOMO transition as well as of the surface states. The emission maximum is at $450 \mathrm{~nm}$ for sample $\mathbf{1}$ and at $430 \mathrm{~nm}$ for $\mathbf{2}$. Sample $\mathbf{1}$ shows an extra emission band at $486 \mathrm{~nm}$ which, based on theoretical predictions, ${ }^{17,18}$ can be related to the higher carboxyl concentration. 
Figure 4 (c) presents the PL of sample 1 at three different temperatures $(330 \mathrm{~K}, 370 \mathrm{~K}$, and $450 \mathrm{~K}$ ). These measurements were carried out after water evaporation from the colloidal suspension of SiC QDs on a clean Si wafer. Compared to figure 4 (a) the dominant component at $330 \mathrm{~K}$ is the emission band situated at $500 \mathrm{~nm}$. Reaching the temperature region where the carboxyl to anhydride reaction and the hydration shell evaporation starts, two bands situated at 410 and $460 \mathrm{~nm}$ are increasing in intensity. At even higher temperature (450 K) it is supposed that the total evaporation of the hydrate shell occurs and the maximum of the emission band shifts to $\sim 400-420 \mathrm{~nm}$. On the other hand, the solvent (water in this case) has a much more important effect on the SiC QDs emission through the surface-solvent interactions. Vytas and coworkers studied the luminescence of $\mathrm{SiC}$ QDs in hydrofluoric acid solution to eliminate the possible oxide layer on the surface and they reported that the emission maximum shifts to lower frequencies ${ }^{40}$ similar as in our study after water evaporation [figure 4 (c)]. Based on the similarities between dry and HF dispersed SiC QDs we conclude that the PL properties of colloidal suspensions of SiC QDs in water depend mainly on the surface structures of $\mathrm{SiC}$ QDs and the water-QD interactions.

\section{Ab initio modeling}

The correlation between the vibrational and optical excitation properties as a function of surface termination was studied by ab initio modeling. First, we investigate the vibrational properties of the $\mathrm{C}=\mathrm{O}$ containing groups. The vibrational properties of individual carboxyl ${ }^{17}$ and carboxylate ${ }^{18}$ groups were already reported that are in good agreement with the experimental findings. We rather focus now on the vibrational properties of the anhydride groups. An anhydride-SiC QD surface bond can form in three different ways (see Fig. 5): by second-neighbor $\mathrm{C}-\mathrm{C}$ (a) or $\mathrm{Si}-\mathrm{Si}$ (b) atoms forming a six-member ring, or it can bond to first-neighbor $\mathrm{Si}-\mathrm{C}$ (c) atoms forming a five-member ring. In the first two cases, the $\mathrm{C}$ or $\mathrm{Si}$ atoms are at (111) facets of the $\mathrm{SiC}$ crystal with about $3.0 \AA$ distance from each other that is almost ideal for the $\mathrm{C}-\mathrm{O}-\mathrm{C}$ bridge of the anhydride group. In the latter, the first neighbor 
Si-C distance is about $1.9 \AA$ that may occur at the edge of (111) and (001) facets of $\mathrm{SiC}$ QDs. The calculated two characteristic vibrational frequencies for configurations a), b) and c) are $(1715,1770),(1702,1770)$ and $(1737,1835) \mathrm{cm}^{-1}$, respectively. While the absolute

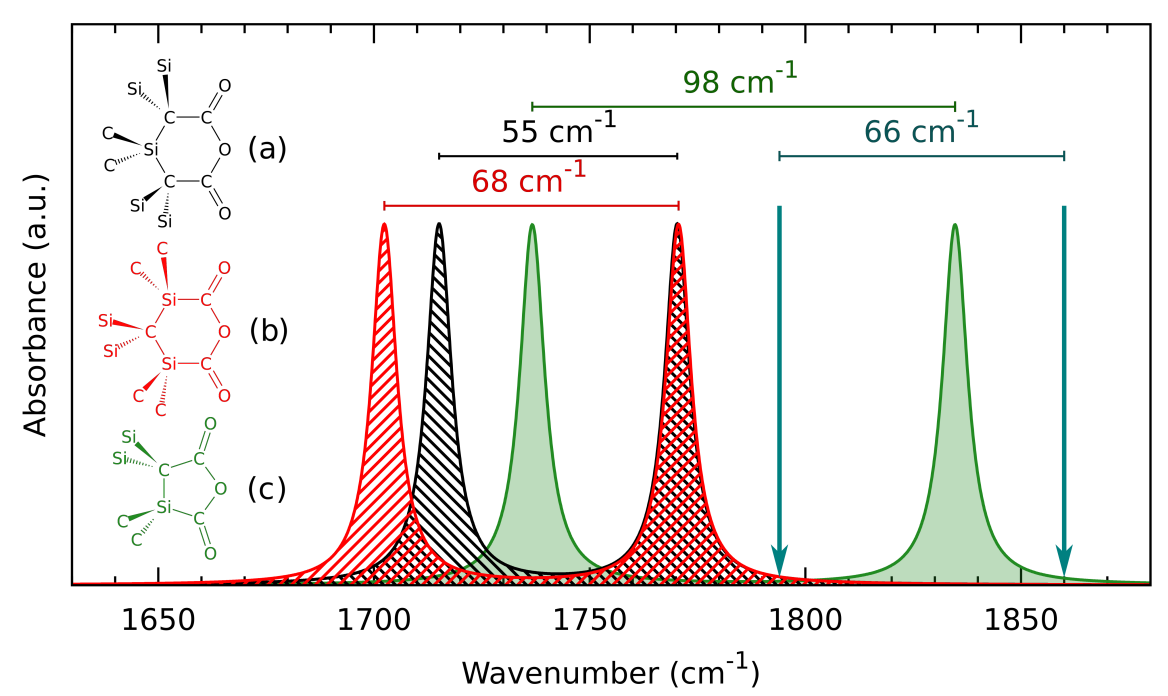

Figure 5: The skeletal formulas and calculated vibrational energies for the three possible anhydride group configurations on the surface of a SiC QD. Black, red and green colors represent anhydride configurations a), b) and c), respectively. We applied an artificial Lorentz broadening of $10 \mathrm{~cm}^{-1}$ for the sake of visibility. The blue vertical arrows mark the positions of the experimentally measured absorption peaks in Fig. 3 (a). The differences between the characteristic vibrational frequencies of the anhydride group are labeled over the horizontal arrows in all the three cases.

values of the calculated frequencies are within $5 \%$ smaller than the experimental ones, it is expected that the chosen methodology is able to well reproduce the relative positions of the two characteristic vibrational modes. This indicates that the five-member ring (c) can be definitely excluded as the origin of the observed IR peaks, as the calculated relative position of $\sim 100 \mathrm{~cm}^{-1}$ is significantly larger than the observed $66 \mathrm{~cm}^{-1}$. This is quite plausible as the number of possible sites for these five-member rings is much smaller than that for the sixmember rings, and the geometry of six-member rings is much less strained. The calculated relative position of the $\mathrm{Si}-\mathrm{Si}$ (b) configuration's vibrational modes is within $0.5 \%$ compared to experiment, while it is within $20 \%$ for the C-C configuration (a). According to our analysis [see inset in Fig. 3 (a)] the two characteristic vibrational modes belong to a single anhydride configuration which implies together with the ab initio results that the anhydride forms on 
the Si side of SiC QDs. Nevertheless, we cannot fully exclude the formation of six-member rings of $\mathrm{C}-\mathrm{C}$ configuration. Still, we continue the analyses on Si-Si configuration and then we discuss the effect on the optical properties of C-C configuration.

We calculated the electronic structure and optical excitation energies of this anhydride and the pair of nearby carboxyl groups that can form the anhydride after dehydration at elevated temperatures on the Si-side of SiC QD surface (see Fig. 6). We assumed that one of the carboxyl groups is deprotonated in aqueous environment that can seriously change the optical properties of the entire SiC QDs. ${ }^{18}$ These carboxylate groups on the surface introduce a defect level in the gap of the pristine SiC QDs [see Fig. 6 (a)], which becomes the new highest occupied molecular orbital (HOMO) of the nanoparticle, while the lowest unoccupied molecular orbital (LUMO) remains the same bulklike state. The anhydride group introduces states outside the gap of pristine $\mathrm{SiC}$ QDs [Fig. 6 (b)]. As a conclusion, the chemical gap opens upon the formation of anhydride groups from carboxylate groups, thus the corresponding excitation energies may also show this trend. We calculated the excitation energies by TDDFT method, and we found that the lowest energy excitation occurs between the HOMO and LUMO at both surface terminations which contributes to the luminescence of SiC nanoparticles. The calculated ZPL wavelengths are $500 \mathrm{~nm}$ and $383 \mathrm{~nm}$ for $\mathrm{SiC}$ nanoparticles with carboxylate and anhydride groups at the surface, respectively. These values are relatively close to the measured maximum intensities in the PL spectra [c.f., Fig. 4(c)], however, this might be fortuitous as the PL spectra were taken at elevated temperates where it is expected that the maximum of the PL emission arises at longer wavelengths than the ZPL wavelength. Nevertheless, it is out of the scope of the paper to deeply analyze the role of electron-vibration coupling in the luminescence of $\mathrm{SiC}$ QDs but we are rather willing to provide a semiquantitative description about the change of the optical properties upon surface modification. To this end, we use the calculated ZPL energies in the analysis. We conclude that the anhydride formation at the surface results in a blueshift of about $117 \mathrm{~nm}$ in the calculated zero-phonon lines. These value is in good agreement with the 


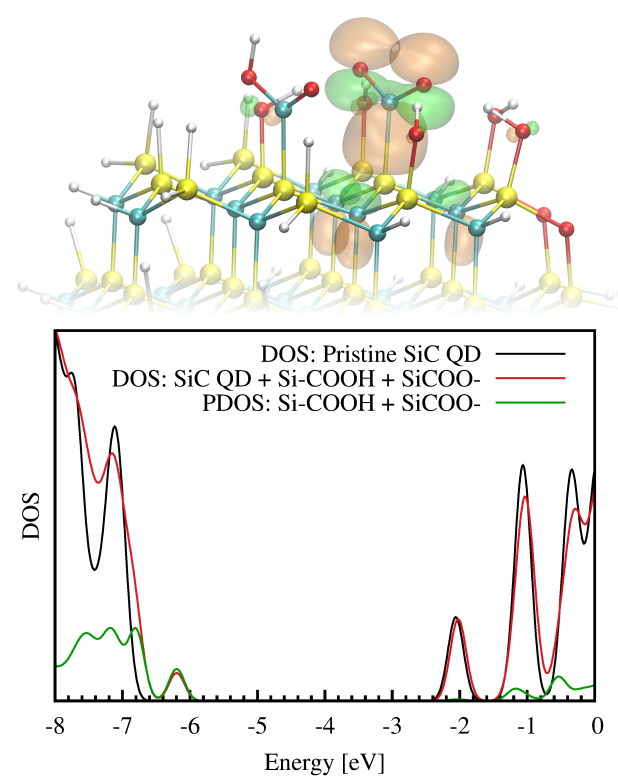

(a)
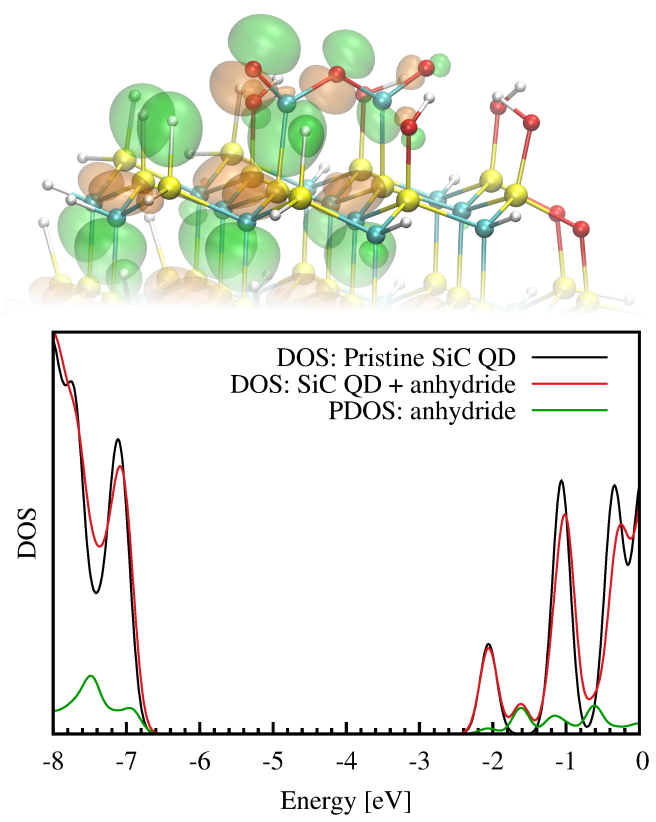

(b)

Figure 6: The HOMO wavefunction, the total (DOS) and projected density of states (PDOS) around the Fermi level for the pair of $\mathrm{COO}^{-}$and $\mathrm{COOH}$ groups (a), and for the anhydride group on the Si side of SiC QDs (b). The PDOS is generated by a projection of the KohnSham orbitals to the atoms of the relevant functional groups in both cases. The $\mathrm{COO}^{-}$ group introduces a defect level localized on the oxygen atoms, while the LUMO remains a delocalized bulklike state in the case of the anhydride group. The PDOS values are multiplied with a factor of 5 for the sake of visibility. An artificial broadening of $0.15 \mathrm{eV}$ is applied for the visualization of DOS and PDOS. White, cyan, yellow and red balls depict H, C, Si and O atoms, respectively. The positive and negative isovalues of the HOMO states are represented by orange and green clouds, respectively. 
experiments [c.f., Fig. 4(c)] where the change in the maximum intensities of the PL peaks is about $100 \mathrm{~nm}$. Thus, the anhydride formation from nearby carboxyl groups at the Si side of the SiC QD surface can be well supported by ab initio modeling. As we did not fully exclude the formation of carboxyl groups on the C-side of the SiC QD surface, we also studied the formation of anhydride groups there, and the change in the optical properties of SiC QDs. The corresponding ZPL energies of $467 \mathrm{~nm}$ and $368 \mathrm{~nm}$ would result in a blueshift of about $89 \mathrm{~nm}$ that is also close to the measured $100 \mathrm{~nm}$. All-in-all, anhydride formation from the pair of carboxyl groups should result in a significant change in the optical properties. This result further proves our previous theoretical assumptions ${ }^{17,18}$ that carboxyl groups play a key role in the optical properties of $\mathrm{SiC}$ QDs in aqueous solution.

\section{Conclusions}

The chemical transformation of carboxyl to acid anhydride groups on the surface of SiC QDs has been studied by multiple methods: synthesis of SiC QDs from two different sources, spectroscopic characterization of the surface structure and luminescent properties, and theoretical calculations. The formation of acid anhydride from carboxyl groups was identified at temperatures around 370-400 K. We consider this anhydride formation an important result as its reactivity is higher compared to carboxyl groups. The discovery of anhydride formation on the $\mathrm{SiC}$ QDs surface allows us to do more simple chemistry for subsequent functionalization and opens new possibilities for further surface engineering steps. We found clear evidence for the presence of hydroxyl termination sites, in accordance with theoretically predicted results. Finally, we assign the redshifts in the PL emission spectra to carboxyl termination and to adsorbed water on the surface of $\mathrm{SiC}$ QDs. 


\section{Acknowledgement}

ZsSz and KK acknowledge the joint project of the Hungarian Scientific Research Fund (OTKA) and the Austrian Science Fund (FWF) under Grant No. ANN 107580. DB acknowledges the support from the European Union and the State of Hungary, co-financed by the European Social Fund in the framework of TÁMOP-4.2.4.A/ 2-11/1-2012-0001 National Excellence Program. AG acknowledges the support from the Hungarian Scientific Fund (OTKA) project Nos. K101819 and K106114, and the Lendület programme of Hungarian Academy of Sciences.

\section{References}

(1) Beke, D.; Szekrényes, Z.; Balogh, I.; Veres, M.; Fazakas, E.; Varga, L. K.; Kamarás, K.; Czigány, Z.; Gali, A. Characterization of Luminescent Silicon Carbide Nanocrystals Prepared by Reactive Bonding and Subsequent Wet Chemical Etching. Appl. Phys. Lett. 2011, 99, 213108.

(2) Beke, D.; Szekrényes, Z.; Balogh, I.; Czigány, Z.; Kamarás, K.; Gali, A. Preparation of Small Silicon Carbide Quantum Dots by Wet Chemical Etching. J. Mat. Res. 2013, 28, 44-49.

(3) Beke, D.; Szekrényes, Z.; Pálfi, D.; Róna, G.; Balogh, I.; Maák, P. A.; Katona, G.; Czigány, Z.; Kamarás, K.; Rózsa, B.; Buday, L.; Vértessy, B.; Gali, A. Silicon Carbide Quantum Dots for Bioimaging. J. Mat. Res. 2013, 28, 205-209.

(4) Mwania, M.; Janáky, C.; Rajeshwar, K.; Kroll, P. Fabrication of $\beta$-SiC Quantum Dots by Photo-Assisted Electrochemical Corrosion of Bulk Powders. Electrochem. Comm. 2013, 37, $1-4$.

(5) Morkoc, H.; Strite, S.; Gao, G. B.; Lin, M. E.; Sverdlov, B.; Burns, M. Large-Band-Gap 
SiC, III-V Nitride, and II-VI ZnSe-Based Semiconductor Device Technologies. J. Appl. Phys. 1994, 76, 1363-1398.

(6) Botsoa, J.; Lysenko, V.; Geloen, A.; Marty, O.; Bluet, J. M.; Guillot, G. Application of 3C-SiC Quantum Dots For Living Cell Imaging. Appl. Phys. Lett. 2008, 92, 173902.

(7) Pourchez, J.; Forest, V.; Boumahdi, N.; Boudard, D.; Tomatis, M.; Fubini, B.; HerlinBoime, N.; Leconte, Y.; Guilhot, B.; Cottier, M.; Grosseau, P. In Vitro Cellular Responses to Silicon Carbide Nanoparticles: Impact of Physico-Chemical Features on Pro-Inflammatory and Pro-Oxidative Effects. J. Nanopart. Res. 2012, 14, 1-12.

(8) Song, H.-J.; Zhang, Z.-Z. Investigation of the Tribological Properties of Polyfluo Wax/Polyurethane Composite Coating Filled with Nano-SiC or Nano-ZrO2. Mater. Sci. Eng. A 2006, 426, $59-65$.

(9) Yakimova, R.; Jr, R. M. P.; Yazdi, G. R.; Vahlberg, C.; Spetz, A. L.; Uvdal, K. Surface Functionalization and Biomedical Applications Based on SiC. J. Phys. D 2007, 40, $6435-6442$.

(10) Maboudian, R.; Carraro, C.; Senesky, D. G.; Roper, C. S. Advances in Silicon Carbide Science and Technology at the Micro- and Nanoscales. J. Vac. Sci. Technol. A 2013, 31, 050805 .

(11) Dai, D.; Guo, X.; Fan, J. Synthesis and Photoluminescence of Semiconductor Quantum Dots/Cetyltrimethylammonium Bromide Vesicle Core/Shell Nanostructures. Appl. Surf. Sci. 2013, 276, $359-362$.

(12) Alekseev, S. A.; Zaitsev, V. N.; Botsoa, J.; Barbier, D. Fourier Transform Infrared Spectroscopy and Temperature-Programmed Desorption Mass Spectrometry Study of Surface Chemistry of Porous 6H-SiC. Chem. Mat. 2007, 19, 2189-2194. 
(13) Fan, J.; Li, H.; Zhang, N.; Lu, R. Identification of the Reconstruction and Bonding Structure of SiC Nanocrystal Surface by Infrared Spectroscopy. Appl. Surf. Sci. 2011, $258,627-630$.

(14) Iijima, M.; Kamiya, H. Surface Modification of Silicon Carbide Nanoparticles by Azo Radical Initiators. J. Phys. Chem. C 2008, 112, 11786-11790.

(15) Li, Y.; Chen, C.; Li, J.-T.; Yang, Y.; Lin, Z.-M. Surface Charges and Optical Characteristic of Colloidal Cubic SiC Nanocrystals. Nanoscale Res. Lett. 2011, 6, 454.

(16) Wu, X. L.; Xiong, S. J.; Zhu, J.; Wang, J.; Shen, J. C.; Chu, P. K. Identification of Surface Structures on 3C-SiC Nanocrystals with Hydrogen and Hydroxyl Bonding by Photoluminescence. Nano Lett. 2009, 9, 4053-4060.

(17) Vörös, M.; Deák, P.; Frauenheim, T.; Gali, A. The Absorption of Oxygenated Silicon Carbide Nanoparticles. J. Chem. Phys. 2010, 133, 064705.

(18) Vörös, M.; Deák, P.; Frauenheim, T.; Gali, A. Influence of Oxygen on the Absorption of Silicon Carbide Nanoparticles. Mater. Sci. Forum 2011, 520, 679-680.

(19) Zakharko, Y.; Botsoa, J.; Alekseev, S.; Lysenko, V.; Bluet, J. M.; Marty, O.; Skryshevsky, V. A.; Guillot, G. Influence of the Interfacial Chemical Environment on the Luminescence of 3C-SiC Nanoparticles. J. Appl. Phys. 2010, 10\%, 013503.

(20) Fan, J. Y.; Wu, X. L.; Li, H. X.; Liu, H. W.; Siu, G. G.; Chu, P. K. Luminescence from Colloidal 3C-SiC Nanocrystals in Different Solvents. Appl. Phys. Lett. 2006, 88, 041909.

(21) Nersisyan, G.; Nikogosov, V.; Kharatyan, S.; Merzhanov, A. Chemical Transformation Mechanism and Combustion Regimes in the System Silicon-Carbon-Fluoroplastic. Combust., Expl., Shock Waves 1991, 27, 720-724. 
(22) Mukasyan, A. S. Combustion Synthesis of Silicon Carbide, Properties and Applications of Silicon Carbide, Prof. Rosario Gerhardt (Ed.); InTech, 2011.

(23) Vörös, M.; Deák, P.; Frauenheim, T.; Gali, A. The Absorption Spectrum of Hydrogenated Silicon Carbide Nanocrystals from Ab Initio Calculations. Appl. Phys. Lett. 2010, 96, 051909.

(24) Perdew, J. P.; Burke, K.; Ernzerhof, M. Generalized Gradient Approximation Made Simple. Phys. Rev. Lett. 1996, 77, 3865-3868.

(25) Blöchl, P. E. Projector Augmented-Wave Method. Phys. Rev. B 1994, 50, 17953.

(26) Kresse, G.; Furthmüller, J. Efficient Iterative Schemes for Ab Initio Total-Energy Calculations Using a Plane-Wave Basis Set. Phys. Rev. B 54, 11169.

(27) Baroni, S.; de Gironcoli, S.; Dal Corso, A.; Giannozzi, P. Phonons and Related Crystal Properties from Density-Functional Perturbation Theory. Rev. Mod. Phys. 2001, 73, $515-562$.

(28) Bauernschmitt, R.; Ahlrichs, R. Treatment of Electronic Excitations within the Adiabatic Approximation of Time Dependent Density Functional Theory. Chem. Phys. Lett. 1996, 256, 454-464.

(29) Somogyi, B.; Zólyomi, V.; Gali, A. Near-Infrared Luminescent Cubic Silicon Carbide Nanocrystals for In Vivo Biomarker Applications: an Ab Initio Study. Nanoscale 2012, 4, $7720-7726$.

(30) Perdew, J. P.; Ernzerhof, M.; Burke, K. Rationale for Mixing Exact Exchange with Density Functional Approximations. J. Chem. Phys. 1996, 105, 9982-9985.

(31) Adamo, C.; Barone, V. Toward Reliable Density Functional Methods Without Adjustable Parameters: The PBE0 Model. J. Chem. Phys. 1999, 110, 6158-6170. 
(32) Jacquemin, D.; Wathelet, V.; Perpéte, E. A.; Adamo, C. Extensive TD-DFT Benchmark: Singlet-Excited States of Organic Molecules. J. Chem. Theory Comput. 2009, $5,2420-2435$.

(33) Harris, D.; Bertolucci, M. Symmetry and Spectroscopy. An Introduction to Vibrational and Electronic Spectroscopy; Dover Publications: New York, 1989.

(34) Somogyi, B.; Gali, A. Computational Design of In Vivo Biomarkers. J. Phys.: Condens. Matter 2014, 26, 143202.

(35) Coates, J. Interpretation of Infrared Spectra, A Practical Approach, in Encyclopedia of Analytical Chemistry; John Wiley and Sons, Ltd, 2006.

(36) Cambaz, G. Z.; Yushin, G. N.; Gogotsi, Y.; Lutsenko, V. G. Anisotropic Etching of SiC Whiskers. Nano Lett. 2006, 6, 548-551.

(37) Eisenberg, A.; Yokoyama, T.; Sambalido, E. Dehydration Kinetics and Glass Transition of Poly(acrylic acid). J. Polym. Sci. Part A-1: Polym. Chem. 1969, 7, 1717-1728.

(38) Szekrényes, Z.; Kamarás, K.; Tarczay, G.; Llannes-Pallas, A.; Marangoni, T.; Prato, M.; Bonifazi, D.; Bjork, J.; Hanke, F.; Persson, M. Melting of Hydrogen Bonds in Uracil Derivatives Probed by Infrared Spectroscopy and Ab Initio Molecular Dynamics. J. Phys. Chem. B 2012, 116, 4626-4633.

(39) Dolenko, T. A.; Burikov, S. A.; Rosenholm, J. M.; Shenderova, O. A.; Vlasov, I. I. Diamond-Water Coupling Effects in Raman and Photoluminescence Spectra of Nanodiamond Colloidal Suspensions. J. Phys. Chem. C 2012, 116, 24314-24319.

(40) Rossi, A. M.; Murphy, T. E.; Reipa, V. Ultraviolet Photoluminescence from 6H Silicon Carbide Nanoparticles. Appl. Phys. Lett. 2008, 92, 253112. 


\section{Graphical TOC Entry}

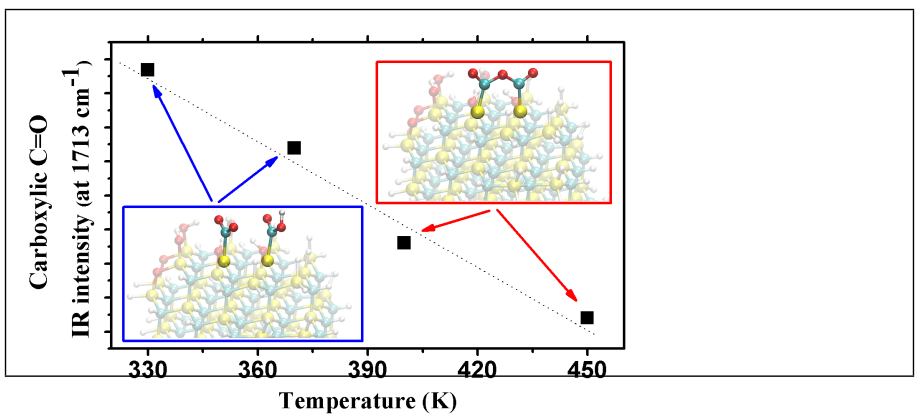

19

20

21

22

23

24

25

26

27

28

29

30

31

32

33

34

35

36

37

38

39

40

41

42

43

44

45

46

47

48

49

50

51

52

53

54

55

56

57

58

59

60 